\title{
Computer-aided design of diffractive beam-shaping elements
}

Thomas Dresel

Thomas Dresel, "Computer-aided design of diffractive beam-shaping elements," Proc. SPIE 3190, Fifth International Topical Meeting on Education and Training in Optics, (8 December 1997); doi: 10.1117/12.294368

Event: Fifth International Topical Meeting on Education and Training in Optics, 1997, Delft, Netherlands 
Computer aided design of diffractive beam-shaping elements

Thomas Dresel

\author{
University of Erlangen, Lehrstuhl für Optik, Staudtstr. 7, D-91058 Erlangen, Germany \\ phone: ++49-9131-858396, fax: ++49-9131- 13508 \\ e-mail: thomas.dresel@pi5wsc.physik.uni-erlangen.de
}

\begin{abstract}
Diffractive beam-shaping elements focus a given aperture with intensity and phase distributions with high efficiency into a pregiven intensity pattern in their focal planes. The design of appropriate phase-only hologram functions can be carried out in a very illustrative and convenient way through the use of geometrical optics. Using inverse raytracing, wavefronts performing geometrical transformations between the hologram and the reconstruction plane can be easily designed. Such geometrical transformations allow to compensate for the intensity and phase distributions of the impinging laser beam as well as for the shape of the hologram aperture. For seperable beam-shaping tasks it is often possible to solve the design problem directly by analytical or numerical integrations. In other cases a numerical approach based on iterative finite element mesh adaption can be used. In this way a variety of elementary reconstruction objects like points, straight line segments, circles, rings, triangles, rectangles etc. in various types of apertures can be handled. More complex reconstruction patterns are decomposed into as few of those elementary objects as possible. The total hologram function is then found by the subsequent superposition of its constituents, with a relative amplitude and phase weighting for each of them. This concept leads to a modular construction kit for diffractive optical elements which on the one hand is easy to use and to understand and on the other hand is a very powerful design tool.
\end{abstract}

Keywords: beam-shaping, diffractive optical element, computer-generated hologram, grating, binary optics

\title{
1. INTRODUCTION
}

One of the major applications of diffractive optical elements is to focus laser beams into two- or threedimensional curves or figures. For many applications the phase distribution along the curves or figures is of no concern. This degree of freedom can be used to optimize the hologram function in terms of diffraction efficiency or simplicity. Usually, iterative Fourier transform algorithms or other nonlinear optimization techniques are used for that purpose. These methods rely on the idea of sampling: the desired reconstruction pattern is regarded to consist of a finite number of discrete point objects, with the phase of each of them as an independent optimization parameter.

An alternative philosopy wich has been proven to be very successful during the last years is the following: a given reconstruction pattern is regarded as consisting of a few elementary objects, such as points, lines, rings, circles, rectangles, etc. For each elementary object there is an appropriate phase-only hologram function that can be derived from geometrical considerations, as described in sections 2 and 3. Finally, the total hologram function is found by the subsequent superposition of the elementary object functions, as described in section 4 .

Assuming the reconstruction pattern can be decomposed into only a few objects, we find that the degrees of freedom are dramatically reduced with those found by regarding the reconstruction pattern as a sampled two- or threedimensional scene. Timeconsuming iterations computing the wave propagation between the hologram and the reconstruction plane can be totally avoided. Moreover, phase functions designed by geometrical optics are smooth what minimizes speckle noise in the optical reconstruction.

Altogether, pure geometrical considerations and the consequent utilization of the superposition principle lead to a very illustrative and powerful design method for diffractive optical beam-shaping elements which is also very well suited for educational purposes. 


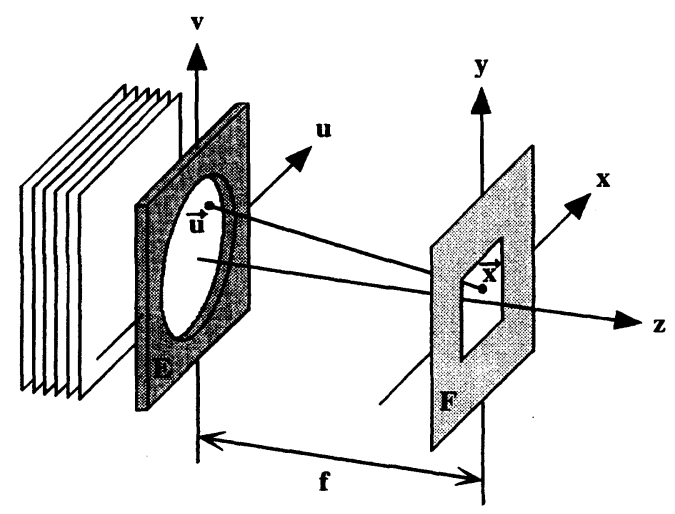

Fig 1. Reconstruction geometry. An incident wave on the aperture plane $E$ is to be focused into a pregiven intensity pattern at the focal plane $F$.

\section{INVERSE RAYTRACING}

For the design of beam-shaping holograms consider the geometry shown in Fig. 1. Coordinates inside the hologram aperture are denoted as $\overrightarrow{\mathrm{u}}=(\mathrm{u}, \mathrm{v})$. An incoming laser beam, characterized by its intensity and phase distributions $\mathrm{I}_{\mathrm{E}}(\overrightarrow{\mathrm{u}})$ and $\phi_{E}(\vec{u})$, respectively, impinges on a phase-only hologram. For the sake of mathematical convenience we write the resulting phase distribution behind the element as $k \psi(\overrightarrow{\mathrm{u}})$ with $\psi$ being the well-known eikonal. Thus, the effect of the hologram can be described by just the addition of the hologram phase $\phi_{\mathrm{H}}(\overrightarrow{\mathrm{u}})$ to the beam phase $\phi_{\mathrm{E}}(\overrightarrow{\mathrm{u}})$ :

$$
\mathrm{k} \Psi(\mathrm{u}, \mathrm{v})=\Phi_{\mathrm{H}}(\mathrm{u}, \mathrm{v})+\Phi_{\mathrm{E}}(\mathrm{u}, \mathrm{v})
$$

In the reconstruction plane $F$, labeled by the coordinates $\overrightarrow{\mathrm{x}}=(\mathrm{x}, \mathrm{y})$ at a distance $\mathrm{f}$ behind the hologram, we want to obtain a specified intensity distribution $\mathrm{I}_{\mathrm{F}}(\overrightarrow{\mathrm{x}})$. To avoid discontinous wave fronts leading to speckle noise we want to obtain a smooth phase function. Hence there must be a smooth coordinate tramsform $\overrightarrow{\mathbf{x}}(\overrightarrow{\mathrm{u}})$ that describes the path of light rays passing through the hologram aperture at $\overrightarrow{\mathrm{u}}$ and the focal (or reconstruction) plane at $\overrightarrow{\mathrm{x}}$. This concept of geometrical transformation has been introduced by Bryngdahl ${ }^{1,2}$. After we have found the coordinate transform $\overrightarrow{\mathbf{x}}(\overrightarrow{\mathrm{u}})$, the eikonal $\psi$ can be retrieved from the slope equation

$$
\vec{\nabla}_{\perp} \Psi(\overrightarrow{\mathrm{u}})=\frac{\overrightarrow{\mathrm{x}}(\overrightarrow{\mathrm{u}})-\overrightarrow{\mathrm{u}}}{\sqrt{\mathrm{f}^{2}+[\overrightarrow{\mathrm{x}}(\overrightarrow{\mathrm{u}})-\overrightarrow{\mathrm{u}}]^{2}}}
$$

which follows immediately from the geometry shown in Fig. 1 . The construction of appropriate transforms $\overrightarrow{\mathrm{x}}(\overrightarrow{\mathrm{u}})$ is based on the fulfilment of two requirements: First we assume the absence of absorption losses, i.e., the total energy must be conserved:

$$
\int_{E} I_{E}(\vec{u}) d^{2} \vec{u}=\int_{F} I_{F}(\vec{x}) d^{2} \vec{x}
$$

Second, mapping $\overrightarrow{\mathrm{x}}(\overrightarrow{\mathrm{u}})$ should redistribute the intensity $\mathrm{I}_{\mathrm{E}}(\overrightarrow{\mathrm{u}})$ of the aperture plane into the desired reconstruction distribution $\mathrm{I}_{\mathrm{F}}(\overrightarrow{\mathrm{x}})$, i.e., we require that

$$
I_{F}(\vec{x}(\vec{u})) d^{2} \vec{x}=I_{E}(\vec{u}) d^{2} \vec{u}
$$

where $d^{2} \vec{x}$ is the image of the area element $d^{2} \vec{u}$. As it is well known from elementary calculus that area elements transform with the determinant of the Jacobi matrix, this requirement can also be formulated as

$$
I_{F}(\vec{x}(\vec{u})) \operatorname{det} \frac{\partial \vec{x}}{\partial \vec{u}}=I_{E}(\vec{u})
$$


Form the application under consideration we know both $I_{F}(\vec{x})$ and $I_{E}(\vec{u})$. Many beam-shaping tasks can be reduced to essentially one-dimensional problems by the separation of variables, for example, focusing a circular or square aperture into a straight line segment. In this case the determinant in Eq. (5) reduces to a one-dimensional derivative that can be integrated either analytically (if possible) or numerically. Some examples of this approach can be found in the references ${ }^{3,4}$.

\section{ITERATIVE FINITE ELEMENT MESH ADAPTION}

For the general task of shaping a laser beam into a certain intensity pattern given on an arbitrary surface in three-dimensional space, such an one-dimensional integration approach is not sufficient. A genuine two-dimensional design approach is needed instead. We use the iterative adaption of finite-element meshes for that purpose. The method is too complicated to be described here in detail, but the basic ideas are as follows: We describe the desired reconstruction area $\mathrm{A}$ by a discrete number of nodal points indexed by row and column index pairs $(i, j)$. These nodes form a net that approximates $A$. The net meshes divide $A$ into finite elements that contain a certain amount of energy. If we are able to construct a second mapping from (i, j) space (i.e., a second net) onto the hologram aperture and if we are, moreover, able to optimize both nets in such a way that corresponding elements contain the same amount of energy, then we have constructed a sampled transform $\overrightarrow{\mathrm{x}}(\overrightarrow{\mathrm{u}})$ by means of $\overrightarrow{\mathrm{x}}_{\mathrm{ij}} \leftrightarrow \overrightarrow{\mathrm{u}}_{\mathrm{ij}}$, fulfilling Eqs. (3) and (4).

For an illustration of this general philosophy consider the task of shaping constant intensity and phase distributions on a circular aperture into a homogenous square distribution at a focal distance $\mathrm{f}$. On the left hand side of Fig. 2 the node numbering for a squre mesh used to describe the reconstruction pattern is shown. With the help of a 4point interpolation algorithm the second initial mapping onto the circular aperture shown in the middle of Fig. 2 has been obtained. After a special iterative optimization the mesh shown on the right hand side has been obtained. This mesh divides a circular aperture into finite quadrilatarels where all of them have approximately the same area. Since both the aperture mesh and the reconstruction mesh are defined over the same region in (i, j) space, we are able to identify corresponding nodes in the two planes.
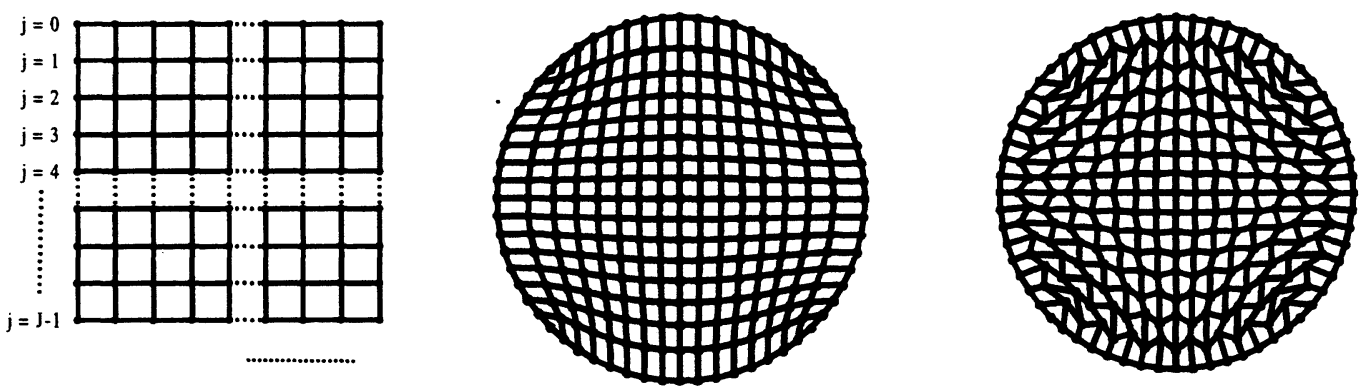

Fig 2: Automatic generation of a mesh mapping from (i, j) space onto a circular aperture by interpolation and subsequent iterative optimization.

Using the slope equation (2) together with the sampled coordinate transform $\overrightarrow{\mathbf{x}}_{\mathrm{ij}} \leftrightarrow \overrightarrow{\mathbf{u}}_{\mathrm{ij}}$, we can immediately determine the partial derivatives at the mesh nodes $(i, j)$. The gradient vector containing the partial derivatives can be regarded as a sampled vector field in $(\mathrm{u}, \mathrm{v})$-space. The problem of reconstructing a wavefront from a measured vector field is very well known in optics: it occurs in analogous form in Shack-Hartmann wavefront sensing and also in shearing interferometry. We assume that $\psi(u, v)$ can be fitted by a finite superposition of Ansatz functions $\Omega_{m}(u, v)$ :

$$
\Psi(u, v)=\sum_{m=1}^{M} a_{m} \Omega_{m}(u, v)
$$

Possible sets of Ansatz functions are cartesian polynomials, Zernike polynomials, Chebyshef polynomials, plane waves etc. Which function set is the most appropriate depends on the symmetry of the vector field to be integrated. Now the desired superposition coefficients $\mathrm{a}_{\mathrm{m}}$ are determined by LSQ-fitting. In this way the desired phase 
distribution can be easily approximated by a few polynomial coefficients which have to be stored. For example, the left side of Fig. 3 shows the real part, i.e. $\cos (k \psi)$, of the wavefront retrieved from the meshes of Figure 2 . The right hand side proves that this wavefront indeed produces approximately the desired reconstruction pattern. It has been obtained by a numerical simulation of the angular spectrum propagation between the hologram and the reconstruction planes and agrees very well with experimental results.
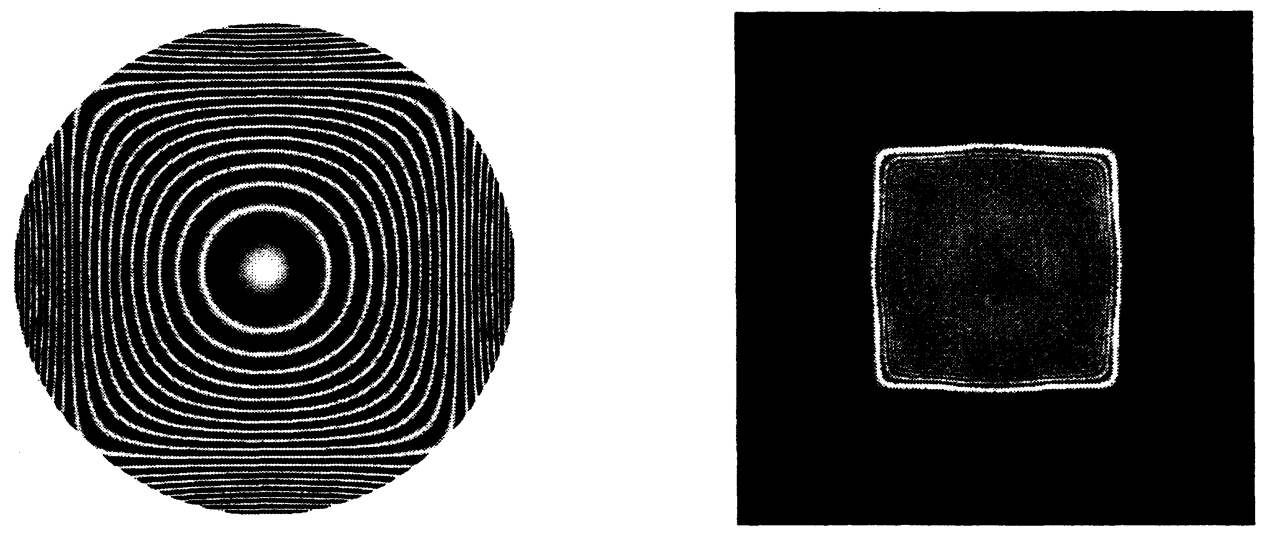

Fig. 3: Left: Real part of a hologram function focusing into a homogeneous square distribtuion, optimized for a circular aperture. Right: Simulated reconstruction.

A second example is shown in fig. 4. Here the square mesh has been mapped onto a slightly deformed square distribution in the reconstruction space. Again the mesh has been optimized with respect to the energy content of the finite elements. As a mapping onto the hologram plane again the mesh of the right hand side of fig. 2 is used. More details about the method of iterative finite element mesh adaption and further design examples can be found in the references ${ }^{5}$.
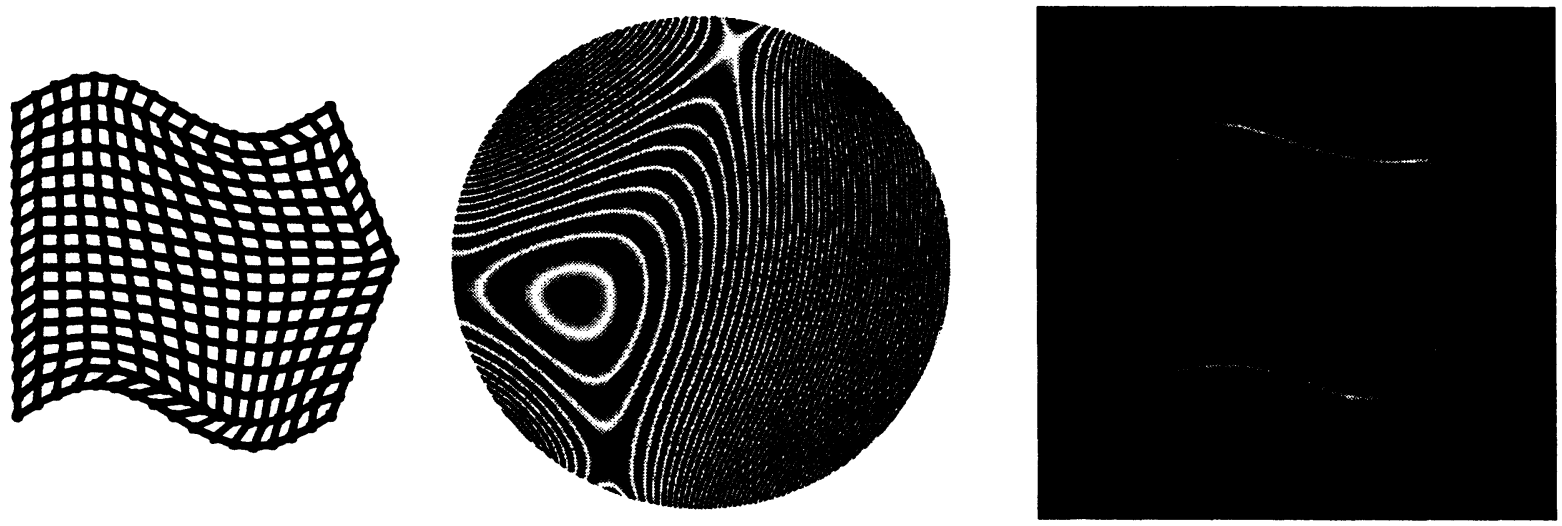

Fig. 4: Left: Mesh mapping describing a slightly deformed square distribution. Middle: Real part of the affiliated hologram function, optimized for a circular aperture. Right: Simulated reconstruction. 


\section{DOE SYNTHESIS}

Composing a hologram of $\mathrm{N}$ elementary objects can be done by simply adding up the complex amplitudes $\exp \left(i \phi_{k}\right), k=1 \ldots N$, where $\phi_{k}$ is the phase function of object $k$. Let $A_{k}$ denote the area covered by object $k$ in the reconstruction plane. In order to achieve a superposition which reconstructs objects of equal intensity, an amplitude weight proportional to the square root of $A_{k}$ has to be introduced for each object. Now the relative intensities of each object can be controlled by the square root of an additional weight $b_{k}$. It is also important to control the phase offset $\alpha_{k}$ of an object to adjust the intensity at points of intersection between different objects. Thus the superposition hologram function can be written as

$$
\mathrm{H}(\overrightarrow{\mathrm{u}}):=\sum_{\mathrm{k}} \sqrt{\mathrm{b}_{\mathrm{k}}} \sqrt{\mathrm{A}_{\mathrm{k}}} \exp \left[\mathrm{i}\left(\phi_{\mathrm{k}}(\overrightarrow{\mathrm{u}})+\alpha_{\mathrm{k}}\right)\right]
$$

In general, this function contains amplitude and phase information. We usually neglect the amplitude of the composed hologram and take just its phase. These phase functions are used for the fabrication of multilevel phase elements with up to 8 phase levels using laser lithography and reactive ion etching ${ }^{4}$. In many cases the decomposition of a given 2D- or 3D-scene into elementary objects is not unique. As a rule of thumb the decomposition with the minimum number of objects should be used.

\section{DESIGN EXAMPLES}

Figure 2 shows one example to demonstrate the capabilities of the superposition method outlined above. A double headed arrow reconstruction pattern is decomposed into 10 line segments. The affiliated complex amplitudes in the hologram plane are superimposed assuming a circular aperture with constant intensity and phase distributions of the incoming laser beam. The amplitude of the resulting hologram function is neglected and the phase distribution is encoded in a 4-level diffractive optical element. On the left hand side of Fig. 5 the central part of the first binary mask layer, in the middle the central part of the second binary mask layer is shown. On the right hand side an optical reconstruction is shown. Further experimental results and details about the elements and the fabrication processes can be found in the references ${ }^{4,5}$.
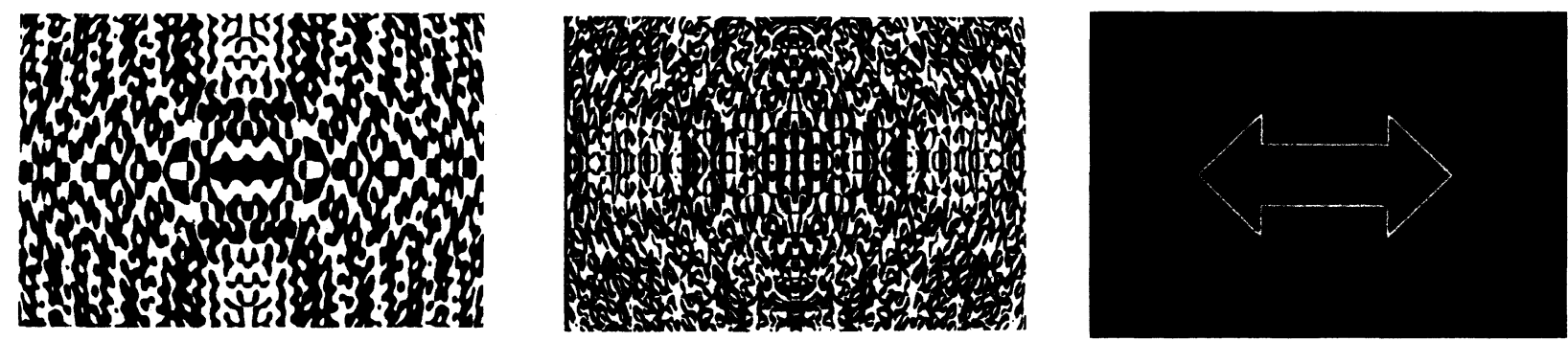

Fig. 5: Left and middle: central parts of the $\pi$ - and the $\pi / 2$-maks layers, respectively, of a diffractive optical element focusing into a double headed arrow. Right: optical reconstruction.

\section{CONCLUSION}

Within the framework of geometrical optics it is possible to trace the task of designing beam-shaping elements back to the task of defining appropriate coordinate transforms between the hologram and the reconstruction plane. These coordinate transforms are defined by analytical or numerical means. Afterwards, smooth phase distributions are retrieved. Wavefronts focussing into different objects can be combined in the hologram plane either by aperture segmentation and/or by superposition of the affiliated complex amplitudes. 
The design of diffractive optical elements according to this approach is not quite as general as the iterative Fourier approach. But on the other hand it has some advantages which make it the superior method for many technical applications. First, sampling of the reconstruction pattern can be totally avoided. The final hologram function is defined continously and thus can be easily adapted to any output grid no matter how coarse or dense it may ever be (with modern lithography systems we talk about billions of pixels!). Second, the degrees of freedom are dramatically reduced compared with those of pure sampling approaches what helps to save computing time and memory. Third, due to the smoothness of the constituent functions, speckle noise in the reconstruction can be minimized. And last but not least we never need to work with random phases. Therefore, the designed diffractive structures resemble the symmetry of the desired reconstruction pattern what makes the design process more transparent and easier to understand.

\section{REFERENCES}

1. O. Bryngdahl, "Optical map transformations", Opt. Comm. 10, 164-168 (1974).

2. O. Bryngdahl, "Geometrical transformations in optics", J. Opt. Soc. Am. 64, 1092-1099 (1974).

3. M. A. Golub, I. N. Sisakyan, V. A. Soifer, "Infrared radiation focusators", Opt. Lasers Eng. 15, 297-309 (1991).

4. T. Dresel, M. Beyerlein, J. Schwider, "Design and fabrication of computer-generated beam-shaping holograms",. Appl. Opt. 35, 4615-4621 (1996).

5. T. Dresel, M. Beyerlein, J. Schwider, "Design of computer-generated beam-shaping holograms by iterative finiteelement mesh adaption", Appl. Opt. 35, 6865-6874 (1996). 